\title{
Multi-dimensional Tensor Sketch: Dimensionality Reduction That Retains Efficient Tensor Operations
}

\author{
Yang Shi ${ }^{1}$ Animashree Anandkumar ${ }^{2}$
}

\begin{abstract}
Sketching refers to a class of randomized dimensionality reduction methods that aim to preserve relevant information in large-scale datasets. They have efficient memory requirements and typically require just a single pass over the dataset. Efficient sketching methods have been derived for vector and matrix-valued datasets. When the datasets are higher-order tensors, a naive approach is to flatten the tensors into vectors or matrices and then sketch them. However, this is inefficient since it ignores the multi-dimensional nature of tensors. In this paper, we propose a novel multidimensional tensor sketch (MTS) that preserves higher order data structures while reducing dimensionality. We build this as an extension to the popular count sketch (CS) and show that it yields an unbiased estimator of the original tensor. We demonstrate significant advantages in compression ratios when the original data has decomposable tensor representations such as the Tucker, CP, tensor train or Kronecker product forms. We apply MTS to tensorized neural networks where we replace fully connected layers with tensor operations. We achieve nearly state of art accuracy with significant compression on image classification benchmarks.
\end{abstract}

\section{Introduction}

Many modern machine learning and data mining applications involve manipulating large-scale multi-dimensional data structures. For instance, the input data can be multimodal or multi-relational (e.g., a combination of image and text), and the intermediate computations can involve higherorder tensor operations (e.g., layers in a tensorized neural network). Memory, bandwidth and computational requirements are usually bottlenecks when these operations are done at scale. Efficient dimensionality reduction schemes

\footnotetext{
${ }^{1}$ University of California, Irvine, USA ${ }^{2}$ California Institute of Technology, USA. Correspondence to: Yang Shi $<$ shiy4@uci.edu $>$.
}

can greatly alleviate this issue if they can find a compact representation while preserving accuracy.

A popular class of dimensionality reduction techniques involves spectral decomposition, such as principle component analysis (PCA), singular value decomposition (SVD) and tensor decompositions. These techniques aim to fit the datasets using low rank approximations, which can be interpreted as a low-dimensional latent structure in the data. They have shown good performance in many applications such as topic modelling (Aza et al. 2001) and recommendation systems (Koren et al., 2009). However, for large-scale datasets, exact computation of spectral decompositions is expensive and randomized methods are used instead.

Sketching forms another class of randomized dimensionality reduction methods. These methods have efficient memory requirements and typically do just a single pass on the data. They have approximation guarantees in recovering the original data. In addition, they allow for certain operations to be accurately carried out in the low-dimensional sketched space, e.g., inner products and outer products on vectors. Count sketch (CS) is a simple sketching technique (Charikar et al. 2002) that uses random signs and random hash functions and has been applied in many settings. For instances, Demaine et al. (2002) design a frequency estimation of internet packet streams. Their objective is to determine essential features of the traffic stream using limited space. Another application is multi-modal pooling of features, e.g., in visual question and answering (VQA), which involves bilinear pooling of image and text features.

Sketching techniques, however, mostly focus on vector and matrix-valued data. A few works attempt to extend to higherorder tensors, e.g., to non-linear kernels (Pham \& Pagh, 2013) and to higher order tensors (Wang et al. 2015), which we refer to as Count-based Tensor Sketch(CTS). However, they view the tensor as sets of vectors and sketch along each fibre ${ }^{1}$ of the tensor. Hence, they inherently still use vector-based sketching techniques and do not exploit the complete multi-dimensional nature of the data. Therefore, correlations across different modes can be missed. As an example, the image features extracted from the traditional

\footnotetext{
${ }^{1} \mathrm{~A}$ fibre is the higher order analogue of a matrix row or column.
} 
computer vision networks often contain spatial information. Separating these data at each location ignores the connection between adjacent pixels.

Main contributions: We propose multi-dimensional tensor sketch (MTS), which is the first sketching to fully exploit the multi-dimensional nature of higher order tensors. It projects the original tensor to another tensor, which can be of the same order, but with smaller dimensions (which affects the recovery guarantees). It allows for efficient computation of various operations such as tensor products and tensor contractions by directly applying operations on the sketched components. MTS has advantages over vectorbased sketching methods such as CTS when the underlying tensor has special forms such as Kronecker product, Tuckerform, CP-form or tensor-train forms. Computation and memory improvement over CTS is shown in Table 1 .

We compare MTS and CTS for Kronecker product compression using synthetic data. MTS outperforms CTS in terms of relative error while using $10 \times$ less computation time while keeping the same compression ratio. Moreover, we apply MTS for approximating tensor operations in tensorized neural networks. These networks replace fully connected layers with multi-linear tensor algebraic operations. This results in compression since multi-linear layers can take better advantage of spatial information available in activations from convolutional layers. Applying MTS to tensor operations results in further compression while preserving accuracy. We demonstrate the efficiency of our approach on the CIFAR10 dataset.

Table 1: Ratio of improvement using MTS over CTS on different tensor operations. Assume the input for the Kronecker product is a $n$-dimension square matrix, and the input for tensor contraction is a third-order tensor with dimension $n$ and rank $r$.

\begin{tabular}{|c|c|c|}
\hline Operator & Computation & Memory \\
\hline Kronecker Product & $O(n)$ & $O\left(n^{2}\right)$ \\
\hline Tucker-form Tensor & $O\left(r^{2}\right)$ if $r^{3}<n, O\left(r^{3}\right)$ if $r^{3}>n$ & $O(r)$ \\
\hline CP-form Tensor & $O(r)$ if $r>n$ & $O(r)$ \\
\hline Tensor-train & $O\left(r^{2}\right)$ if $\log r>n$ & $O(n)$ \\
\hline
\end{tabular}

Important Tensor Applications: We focus on tensor sketching because data is inherently multi-dimensional. In probabilistic model analysis, tensor decomposition is the crux of model estimation via the method of moments. A variety of models such as topic models, hidden Markov models, Gaussian mixtures etc., can be efficiently solved via the tensor decomposition techniques under certain mild assumptions (Anandkumar et al., 2014). Tensor methods are also relevant in deep learning. Yu et al. learn the nonlinear dynamics in recurrent neural networks directly using higher order state transition functions through tensor train decomposition. Kossaifi et al. (2017) propose tensor contraction

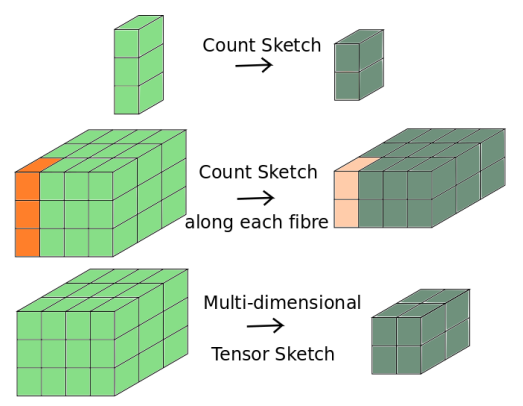

Figure 1: Multi-dimensional tensor sketch of a third-order tensor.

Table 2: Summary of notations.

\begin{tabular}{|c|c|}
\hline Notation & Meaning \\
\hline FFT(a) & 1D Fourier Transform \\
\hline IFFT(a) & 1D Inverse Fourier Transform \\
\hline$a \circ b$ & Element-wise Product \\
\hline$a * b$ & Convolution \\
\hline$A \otimes B$ & Kronecker Product \\
\hline FFT2(A) & 2D Fourier Transform \\
\hline IFFT2(A) & 2D Inverse Fourier Transform \\
\hline$A^{-1}$ & Matrix Inverse \\
\hline$\hat{\mathcal{A}}$ & Decompression \\
\hline$[\mathrm{n}]$ & $\{1,2, \cdots, n\}$ \\
\hline
\end{tabular}

and regression layers in deep convolutional neural networks. Instead of mapping high-order activation tensor to vectors to pass through a fully connected layer, they generate tensor weights to filter the multi-dimensional activation tensor. Moreover, the tensor product is used to combine different features in multi-modal tasks. Visual Question Answering task Antol et al. 2015) requires integration of feature maps from image and text that have drastically different structures. Many studies have been conducted to explore various features and combine them wisely based on their structures(Fukui et al., 2016, Teney et al., 2017, Shi et al., 2018).

\section{Multi-dimensional Tensor Sketch}

In this section, we first present important definitions and theorems defined in the previous literature. We then outline the multi-dimensional tensor sketch algorithm and present its approximation guarantees. We show the superiority of this sketch, comparing to CS, in approximating Kronecker product.

\subsection{Tensor}

We denote vectors by lowercase letters, matrices by uppercase letters, and higher-order tensors(multi-dimensional data structures) by calligraphic uppercase letters. The order 


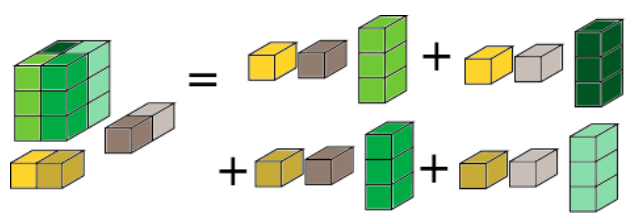

Figure 2: Tensor contraction example: given $\mathcal{A} \in \mathbb{R}^{2 \times 2 \times 3}$, $u \in \mathbb{R}^{2 \times 1}, v \in \mathbb{R}^{2 \times 1}, \mathcal{A}(u, v, I) \in \mathbb{R}^{1 \times 1 \times 3}\left(I \in \mathbb{R}^{3 \times 3}\right.$ is an identity matrix).

of a tensor is the number of modes it admits. For example, $\mathcal{T} \in \mathbb{R}^{n_{1} \times \cdots \times n_{N}}$ is an $N$ th-order tensor because it has $\mathrm{N}$ modes. A scalar is a zeroth-order tensor, a vector is a firstorder tensor, a matrix is a second-order tensor with the rows being the first mode and columns being the second mode, and a three-way array ( say $\mathcal{A} \in \mathbb{R}^{m \times n \times p}$ ) is a third-order tensor with the first, second and third modes indexed by $m$, $n$, and $p$, respectively.

Tensor product is known as outer product in vectors case. An $N$ th-order tensor is an element of the tensor product of $N$ vector spaces: $\mathcal{A}=v_{1} \otimes \cdots \otimes v_{N} \in$ $\mathbb{R}^{n_{1} \times \cdots \times n_{N}}$, where $v_{i} \in \mathbb{R}^{n_{i}}, i \in 1, \ldots, N$. Assume $V_{i} \in \mathbb{R}^{n_{i} \times m_{i}}, i \in 1, \ldots, N$, then tensor contraction is denoted as $\mathcal{A}\left(V_{1}, \cdots V_{N}\right) \in \mathbb{R}^{m_{1} \times \cdots \times m_{N}}$, and defined element-wise as:

$\mathcal{A}\left(V_{1}, \ldots, V_{N}\right)_{i_{1}, \ldots, i_{N}}=\sum_{j_{1} \cdots j_{N}} \mathcal{A}_{j_{1}, \ldots, j_{N}} V_{1 j_{1}, i_{1}} \cdots V_{N j_{N}, i_{N}}$

We show a tensor contraction example in Figure 2. Tensor decomposition is an extension of matrix decomposition to higher orders. The Tucker decomposition (Tucker. 1966) is analogous to principal component analysis. It decomposes a tensor as a core tensor contracted with a matrix along each mode. For instance, a third-order tensor $\mathcal{T} \in \mathbb{R}^{n_{1} \times n_{2} \times n_{3}}$ has the Tucker decomposition:

$$
\mathcal{T}=\mathcal{G}(U, V, W)
$$

where $\mathcal{G} \in \mathbb{R}^{r_{1} \times r_{2} \times r_{3}}, U \in \mathbb{R}^{n_{1} \times r_{1}}, V \in \mathbb{R}^{n_{2} \times r_{2}}, W \in$ $\mathbb{R}^{n_{3} \times r_{3}}$. CANDECOMP/PARAFAC(CP) (Harshman, 1970) is a special case of a Tucker-form tensor, where the core tensor is a sparse tensor that only has non-zero values on the superdiagnoal. It can be represented as a sum of rank-1 tensors. In previous example, $\mathcal{T}=\sum_{i=1}^{r} \mathcal{G}_{i i i} U_{i} \otimes V_{i} \otimes$ $W_{i}$. Figure 3 shows the Tucker-form and the CP-form of a third-order tensor. By splitting variables, the Tensor Train (TT) (Oseledets, 2011) can represent a higher-order tensor by several third-order tensors.

\subsection{Count Sketch}

We describe Count Sketch(CS) in Algorithm 1, The estimation can be made more robust by taking $d$ independent sketches of the input and calculating the median of the $d$
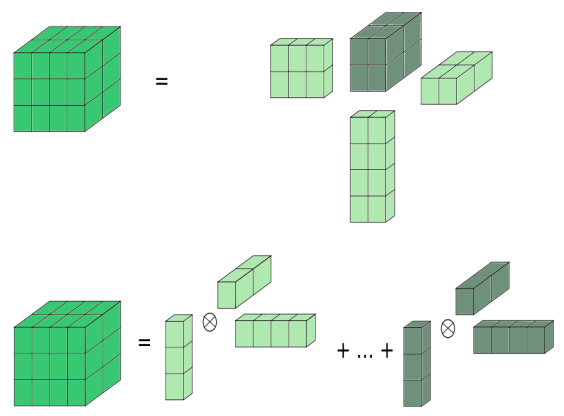

Figure 3: Tucker(upper) and $\mathrm{CP}($ lower) decomposition of a third-order tensor.

estimators. Charikar et al. (2002) prove that the CS is an unbiased estimator with variance bounded by the 2-norm of the input. Pagh (2012) use CS and propose a fast algorithm to compute count sketch of an outer product of two vectors.

$$
\mathrm{CS}(u \otimes v)=\mathrm{CS}(u) * \operatorname{CS}(v)
$$

The convolution operation can be transferred to elementwise product using FFT properties. Thus, the computation complexity reduces from $O\left(n^{2}\right)$ to $O(n+c \log c)$, if the vectors are of size $n$ and the sketching size is $c$.

Note that CS can be applied to each fibre of the input tensor, we can always sketch a tensor using CS. We call this Count-based Tensor Sketch(CTS). Algorithm 2 describes the CTS. The disadvantage is the ignorance of the connections between fibres, as we mentioned in Section 1 .

\subsection{Proposed: Multi-dimensional Tensor Sketch(MTS)}

Given a tensor $\mathcal{T} \in \mathbb{R}^{n_{1} \times \cdots \times n_{N}}$, random hash functions $h_{k}:\left[n_{k}\right] \rightarrow\left[m_{k}\right], k \in[N]$, and random sign functions $s_{k}:\left[n_{k}\right] \rightarrow\{ \pm 1\}, k \in[N]$, MTS computes:

$$
\operatorname{MTS}(\mathcal{T})_{\substack{t_{1}, \cdots, t_{N} \\ h_{1}\left(i_{1}\right)=t_{1}, \ldots, h_{N}\left(i_{N}\right)=t_{N}}} s_{1}\left(i_{1}\right) \cdots s_{N}\left(i_{N}\right) \mathcal{T}_{i_{1}, \ldots, i_{N}}
$$

Using tensor operations, we find the compact formulation

$$
\operatorname{MTS}(\mathcal{T})=(\mathcal{S} \circ \mathcal{T})\left(H_{1}, \ldots, H_{N}\right)
$$

Here, $\mathcal{S}=s_{1} \otimes \cdots \otimes s_{N} \in \mathbb{R}^{n_{1} \times \cdots \times n_{N}}, H_{i} \in \mathbb{R}^{n_{i} \times m_{i}}$, $H_{i}(a, b)=1$, if $h_{i}(a)=b$, for $\forall a \in\left[n_{i}\right]$, otherwise $H_{i}(a, b)=0 . \operatorname{MTS}(\mathcal{T}) \in \mathbb{R}^{m_{1} \times \cdots \times m_{N}}$ equals the signed tensor $(S \circ \mathcal{T})$ contract with the hash matrices $\left(H_{i}\right.$, for $i \in[N]$ ) along each mode. To recover the original tensor, we have

$$
\hat{\mathcal{T}}_{i_{1}, \cdots, i_{N}}=s_{1}\left(i_{1}\right) \cdots s_{N}\left(i_{N}\right) \operatorname{MTS}(\mathcal{T})_{h_{1}\left(i_{1}\right), \cdots, h_{N}\left(i_{N}\right)}
$$

, or in compact format

$$
\hat{\mathcal{T}}=(\mathcal{S} \circ \operatorname{MTS}(\mathcal{T}))\left(H_{1}^{-1}, \cdots, H_{N}^{-1}\right)
$$


We present MTS in Algorithm 3 . We prove that this MTS is unbiased and it has variance of roughly $\|\mathcal{T}\|_{F}$. The detailed proofs are given in Appendix B.2.
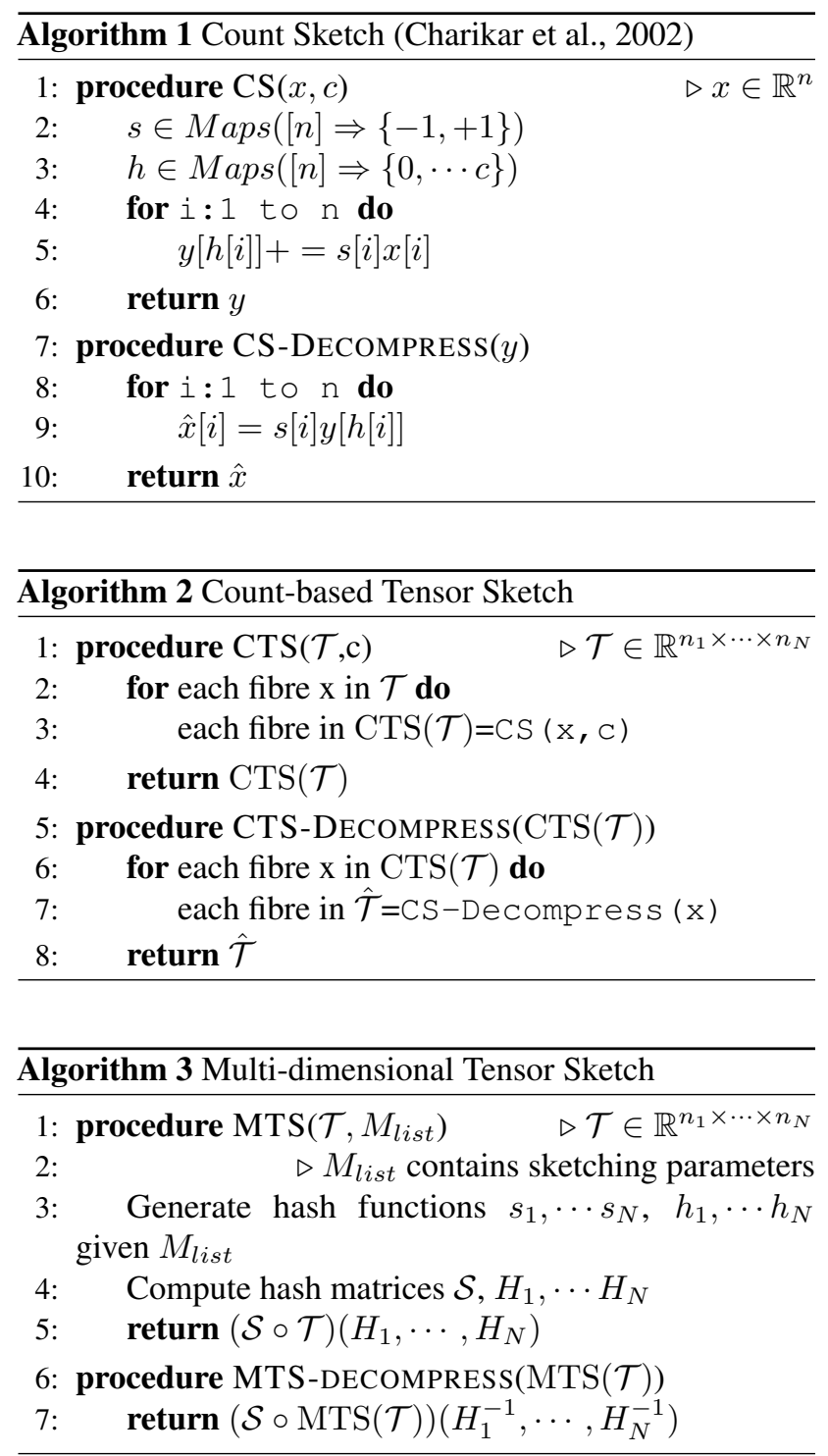

Theorem 2.1 (MTS recovery analysis). Given a tensor $\mathcal{T} \in \mathbb{R}^{n_{1} \times \cdots \times n_{N}}$ and the MTS hash functions $s_{k}, h_{k}$ with sketching dimensions $m_{k}$, for $k \in[N]$, the decompress Equation 4 computes an unbiased estimator for $\mathcal{T}_{i_{1}^{*} \cdots i_{N}^{*}}$ with variance bounded by $\|\mathcal{T}\|_{F}^{2} /\left(m_{1} \cdots m_{N}\right)$, for any $i_{1}^{*}, \cdots, i_{N}^{*}$.

In computational analysis, we assume $\operatorname{MTS}(\mathcal{T})$ takes $O\left(n_{1} \cdots n_{N}\right)$ operations if $\mathcal{T} \in \mathbb{R}^{n_{1} \times \cdots \times n_{N}}$. However, in practise, we need to compute Equation 3 in a sequential way. Without computation optimization this results in unnecessary permutation and copy overheads. For example, given $\mathcal{T} \in \mathbb{R}^{n_{1} \times n_{2} \times n_{3}}, H_{2} \in \mathbb{R}^{n_{2} \times m_{2}}, \mathcal{T}\left(I, H_{2}, I\right)$ can be computed in three steps:
- reshape $\mathcal{T} \in \mathbb{R}^{n_{1} \times n_{2} \times n_{3}}$ to $\mathcal{T} \in \mathbb{R}^{n_{1} n_{3} \times n_{2}}$

- compute $\mathcal{P}=\mathcal{T} H_{2} \in \mathbb{R}^{n_{1} n_{3} \times m_{2}}$

- reshape $\mathcal{P} \in \mathbb{R}^{n_{1} n_{3} \times m_{2}}$ to $\mathcal{P} \in \mathbb{R}^{n_{1} \times m_{2} \times n_{3}}$

Shi et al. (2016) propose a parallel computing scheme for tensor contractions using Basic Linear Algebra Subroutines(BLAS). Based on that, $\mathcal{T}\left(I, H_{2}, I\right)$ can be computed in one step without permutations:

- parallel compute $n_{3}$ number of matrix-matrix multiplications: $\mathcal{P}_{:,, i}=\mathcal{T}_{:,, i} H_{2}, i \in\left[n_{3}\right]$

The recently released High-Performance Tensor Transposition (HPTT) is an open-source library that performs efficient tensor contractions (Springer et al., 2017). By applying these tensor contraction primitives, we can accelerate the sketching process in practice.

\subsection{Example: Approximate Kronecker Product}

Pagh (2012) shows that the count sketch of an outer product equals the convolution between the count sketch of each input vector. Furthermore, the convolution operation in time domain can be transferred to element-wise product in frequency domain. The Kronecker product is a generalization of the outer product from vectors to matrices. It is one kind of a tensor product. Usually, the computation and memory cost are expensive as illustrated in Figure 4

Sketching a Kronecker product using count-based tensor sketch (CTS) We compute the sketch of $A \otimes B$ by sketching each of the outer products(row-wise) using CTS. For $A, B \in$ $\mathbb{R}^{n \times n}$, each outer product requires $O(n+c \log c)$ operations and there are $O\left(n^{2}\right)$ such outer products. Thus, it requires a total $O\left(n^{3}+n^{2} c \log c\right)$ operations to sketch the Kronecker product.

Sketching a Kronecker product using multidimensional tensor sketch (MTS) We show that

$$
\operatorname{MTS}(A \otimes B)=\operatorname{MTS}(A) * \operatorname{MTS}(B)
$$

The convolution in Equation 5 can be further simplified by converting sketched components to the $2 \mathrm{D}$-frequency domain. Assume $A^{\prime}=\operatorname{MTS}(A)$ and $B^{\prime}=\operatorname{MTS}(B)$ :

$$
\operatorname{FFT} 2\left(A^{\prime} * B^{\prime}\right)=\operatorname{FFT} 2\left(A^{\prime}\right) \circ \operatorname{FFT} 2\left(B^{\prime}\right)
$$

This approximation requires $O\left(n^{2}\right)$ to complete MTS(A), MTS(B) and $O\left(m^{2} \log m\right)$ to complete 2D Fourier Transform if the MTS sketching parameter is $m$. The proof for Equation 5 is given in Appendix B.1. This example shows the advantage of MTS in estimating tensor operations by directly applying operations on the sketched components. See Figure 5, 6 and Table 3 for comparison. 

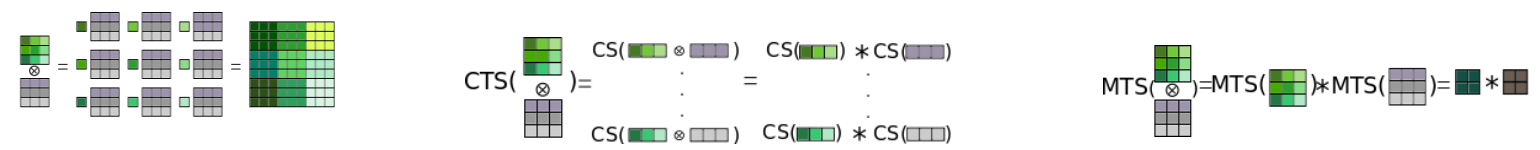

Figure 4: Kronecker product of Figure 5: CTS of Kronecker product. It reFigure 6: MTS of Kronecker product. It two matrices with size $n \times n$. It quires $O\left(n^{2}(n+c \log c)\right)$ computations. requires $O\left(n^{2}+m^{2} \log m\right)$ computations. requires $O\left(n^{4}\right)$ computation.

Table 3: Computation analysis of sketched Kronecker product using MTS and CTS (We choose $O\left(m^{2}\right)=O(c)$ to maintain same recovery error).

\begin{tabular}{|c|c|}
\hline Operator & Computation \\
\hline $\mathrm{CS}(u \otimes v)$ & $O(n+c \log c)$ \\
\hline $\mathrm{CTS}(A \otimes B)$ & $O\left(n^{2}(n+c \log c)\right)$ \\
\hline $\mathrm{MTS}(A \otimes B)$ & $O\left(n^{2}+c \log c\right)$ \\
\hline
\end{tabular}



Figure 7: Sketch of a third-order Tucker-form tensor.

\section{Sketched Tensor Representations}

In this section, we discuss how to approximate tensors given different tensor representations: Tucker-form tensor, CPform tensor and Tensor-train. We show that MTS is more efficient for compressing tensors given these forms compared to CTS, especially when the tensors are high-rank.

\subsection{Tucker-form Tensor Sketching: CTS vs. MTS}

The Tucker decomposition is a form of higher-order PCA. In the following analysis, we use a third-order tensor as an example. Define a tensor $\mathcal{T}=\mathcal{G}(U, V, W) \in \mathbb{R}^{n_{1} \times n_{2} \times n_{3}}$, where $U \in \mathbb{R}^{n_{1} \times r_{1}}, V \in \mathbb{R}^{n_{2} \times r_{2}}, W \in \mathbb{R}^{n_{3} \times r_{3}}$, $\mathcal{G} \in \mathbb{R}^{r_{1} \times r_{2} \times r_{3}}$. To simplify the analysis, we assume $r_{1}=r_{2}=r_{3}=r, n_{1}=n_{2}=n_{3}=n$. Figure 7 shows the difference between the sketching methods using CTS and MTS.

\subsubsection{CTS OF A TUCKER-FORM TENSOR}

To apply count sketch to a Tucker-form tensor, we rewrite the decomposition as a sum of rank-1 tensors. The CTS representation is:

$$
\begin{aligned}
\operatorname{CTS}(\mathcal{T}) & =\sum_{a=1}^{r} \sum_{b=1}^{r} \sum_{c=1}^{r} \mathcal{G}_{a b c} \operatorname{CS}\left(U_{a} \otimes V_{b} \otimes W_{c}\right) \\
& \left.=\sum_{a=1}^{r} \sum_{b=1}^{r} \sum_{c=1}^{r} \mathcal{G}_{a b c} \operatorname{CS}\left(U_{a}\right) * \operatorname{CS}\left(V_{b}\right) * \operatorname{CS}\left(W_{c}\right)\right)
\end{aligned}
$$

where $U_{a}, V_{b}, W_{c}$ are $a^{t h}, b^{t h}, c^{t h}$ column of $U, V, W$ respectively. $\otimes$ is the tensor product(outer product in vector space). In this way, we can apply outer product sketching (Pagh 2012) to each term in the summation. Notice that the convolution operation in Equation 7 can be computed through FFT. The computation complexity of this estimation process is $O\left(r^{3}(n+c \log c)\right)$. The memory complexity is $O\left(r c+r^{3}\right)$.

Suppose $\hat{\mathcal{T}}$ is the recovered tensor for $\mathcal{T}=\mathcal{G}(U, V, W)$ after applying count sketch on $U, V$ and $W$ with sketching dimension $c, g=\|\mathcal{G}\|_{\max }, g_{1}=\max \left(\left\|U_{i}\right\|_{2}\right), g_{2}=$ $\max \left(\left\|V_{j}\right\|_{2}\right), g_{3}=\max \left(\left\|W_{k}\right\|_{2}\right)$ for $i \in[r], j \in[r], k \in$ $[r]$. We suppose the estimation takes $d$ independent sketches of $U, V$ and $W$ and then report the median of the $d$ estimates.

Theorem 3.1 (CTS recovery analysis for Tucker tensor). If $d=\Omega(\log (1 / \delta)), c=\Omega\left(\frac{r^{3} g g_{1}^{2} g_{2}^{2} g_{3}^{2}}{\epsilon^{2}}\right)$, then with probability $\geq 1-\delta$ there is $\left|\hat{\mathcal{T}}_{i j k}-\mathcal{T}_{i j k}\right| \leq \epsilon$.

Theorem 3.1 shows that the sketch length $c$ can be set as $O\left(r^{3}\right)$ to provably approximate a 3rd-order Tucker-form tensor with dimension $n$ and rank $r$.

\subsubsection{MTS OF A TUCKER-FORM TENSOR}

The MTS of a Tucker-form tensor can be accomplished by peforming MTS on each of the factors and the core tensor. We rewrite $\mathcal{T}=(U \otimes V \otimes W) \operatorname{vec}(\mathcal{G})$. Thus,

$$
\begin{aligned}
\operatorname{MTS}(\mathcal{T}) & =\operatorname{MTS}((U \otimes V \otimes W) \operatorname{vec}(\mathcal{G})) \\
& =\sum_{i=1}^{m_{2}} \operatorname{MTS}\left((U \otimes V \otimes W)_{i} \otimes \operatorname{vec}(\mathcal{G})_{i}\right) \\
& =\sum_{i=1}^{m_{2}} \operatorname{MTS}(U \otimes V \otimes W)_{i} * \operatorname{MTS}(\operatorname{vec}(\mathcal{G}))_{i}
\end{aligned}
$$

In (Pagh, 2012), the compressed matrix multiplication is a sum of CS of outer products. Instead of using CS of 
each row/column of the input matrices to compute the approximated outer product, we use each row/column of the multi-dimensional tensor sketched matrices. Notice that since $\mathcal{G}$ does not have any underlying structure, $\operatorname{MTS}(\mathcal{G})=$ $\operatorname{MTS}(\operatorname{vec}(\mathcal{G}))=\operatorname{CS}(\operatorname{vec}(\mathcal{G}))$. The computation complexity of this estimation process is $O\left(n r+m_{1} m_{2} \log \left(m_{1} m_{2}\right)\right)$. The memory complexity is $O\left(m_{1} m_{2}\right)$.

Suppose $\hat{\mathcal{T}}$ is the recovered tensor for $\mathcal{T}=\mathcal{G}(U, V, W)$ after applying MTS on $U, V$ and $W$ with sketching dimension $m_{1}$ and $m_{2}$ along two modes and count sketch on $\mathcal{G}$ with sketching dimension $m_{2}$. Suppose the estimation takes $d$ independent sketches of $\mathcal{G}, U, V$ and $W$ and then report the median of the $d$ estimates:

Theorem 3.2 (MTS recovery analysis for Tucker tensor). If $d=\Omega(\log (1 / \delta))$ and $m_{1} m_{2}=$ $\Omega\left(\frac{r^{3}\|G\|_{F}^{2}\|U\|_{F}^{2}\|V\|_{F}^{2}\|W\|_{F}^{2}}{\epsilon^{2}}\right)$, then with probability $\geq 1-\delta$ there is $\left|\hat{\mathcal{T}}_{i j k}-\mathcal{T}_{i j k}\right| \leq \epsilon$.

Theorem 3.2 shows that the product of sketch length $m_{1} m_{2}$ can be set as $O\left(r^{3}\right)$ to provably approximate a 3rd-order Tucker-form tensor with dimension $n$, rank $r$. Given Theorem 3.1 and 3.2 , if we set $c=O\left(r^{3}\right)$ and $m_{1} m_{2}=O\left(r^{3}\right)$, the estimation error for CTS and MTS will be at the same level. Detailed proofs for Theorem 3.1. Theorem 3.2 are in Appendix B.3

REMARKS: CP-form Tensor Sketching A CP-form tensor can be treated as a special case of a Tucker-form tensor. For a third-order tensor: its core tensor is a sparse tensor, where only the diagonal data has non-zero values. We can write the decomposition as $\mathcal{T}=\sum_{i=1}^{r} \mathcal{G}_{i i i} U_{i} \otimes V_{i} \otimes W_{i}$. We can sketch it in the same way as we described above. The only difference is that instead of summing over all $\mathcal{G}$ values, we sum over only $r$ number of $\mathcal{G}$ values. We summarize the computation and memory analysis in Tables 4 and 5. By performing MTS on the CP-form tensor components, we get a ratio of $O(r)$ improvement if the tensor is overcomplete $(r>n)$.

\subsubsection{Computation And Memory Analysis}

The computation and memory cost is analyzed in Table 5 . We get a computation cost improvement with a ratio of $O\left(r^{2}\right)$ if $r^{3}<n, O\left(r^{3}\right)$ if $r^{3}>n$, compared to the previous CTS method for a Tucker-form Tensor.

\subsection{Tensor-Train Sketching: CTS vs. MTS}

The Tensor-train(TT) decomposition is another important tensor structure. For simplicity, we use a third-order tensor as an example. Assume $\mathcal{T} \in \mathbb{R}^{n_{1} \times n_{2} \times n_{3}}, G_{1} \in \mathbb{R}^{n_{1} \times r_{1}}$, $G_{2} \in \mathbb{R}^{n_{2} \times r_{1} \times r_{2}}, G_{3} \in \mathbb{R}^{n_{3} \times r_{2} \times 1}$, the TT format is: $\mathcal{T}_{i_{1} i_{2} i_{3}}=G_{1}\left[i_{1},::::\right] G_{2}\left[i_{2},::::\right] G_{3}\left[i_{3},::::\right], \forall i_{1} \in\left[n_{1}\right], i_{2} \in$ $\left[n_{2}\right], i_{3} \in\left[n_{3}\right]$. By sketching $G_{1}, G_{2}$ and $G_{3}$, we ob-
Table 4: Computation and memory analysis for Tucker/CP tensor sketching before substituting $c, m_{1}, m_{2}$ with known parameters.

\begin{tabular}{|c|c|c|}
\hline Operator & Computation & Memory \\
\hline \multicolumn{3}{|c|}{ Tucker } \\
\hline $\mathrm{CTS}(\mathcal{T})$ & $O\left(n r^{3}+c r^{3} \log c\right)$ & $O\left(c r+r^{3}\right)$ \\
\hline $\operatorname{MTS}(\mathcal{T})$ & $O\left(n r+r^{3}+m_{1} m_{2} \log \left(m_{1} m_{2}\right)\right)$ & $O\left(m_{1} m_{2}\right)$ \\
\hline \multicolumn{3}{|c|}{ CP } \\
\hline $\operatorname{CTS}(\mathcal{T})$ & $O(n r+c r \log c)$ & $O(c r+r)$ \\
\hline $\operatorname{MTS}(\mathcal{T})$ & $O\left(n r+r+m_{1} m_{2} \log \left(m_{1} m_{2}\right)\right)$ & $O\left(m_{1} m_{2}\right)$ \\
\hline
\end{tabular}

Table 5: Computation and memory analysis for Tucker/CP tensor sketching after substituting $c, m_{1}, m_{2}$ with known parameters (keep both methods with same recovery error).

\begin{tabular}{|c|c|c|}
\hline Operator & Computation & Memory \\
\hline \multicolumn{3}{|c|}{ Tucker } \\
\hline $\mathcal{T}$ & $O\left(n^{3} r+n^{2} r^{2}+n r^{3}\right)$ & $O\left(n r+r^{3}\right)$ \\
\hline $\operatorname{CTS}(\mathcal{T})$ & $O\left(n r^{3}+r^{6} \log r\right)$ & $O\left(r^{4}\right)$ \\
\hline $\operatorname{MTS}(\mathcal{T})$ & $O\left(n r+r^{3} \log r\right)$ & $O\left(r^{3}\right)$ \\
\hline \multicolumn{3}{|c|}{ CP } \\
\hline $\mathcal{T}$ & $O\left(n^{3} r^{3}\right)$ & $O(n r+r)$ \\
\hline $\operatorname{CTS}(\mathcal{T})$ & $O\left(n r+r^{2} \log r\right)$ & $O\left(r^{2}\right)$ \\
\hline $\operatorname{MTS}(\mathcal{T})$ & $O(n r+r \log r)$ & $O(r)$ \\
\hline
\end{tabular}

tain an estimation of the TT format tensor. We assume $n_{1}=n_{2}=n_{3}=n, r_{1}=r_{2}=r$.

- CTS Count sketch is performed along the fibers of size $n$ with sketching dimension $c$. In other words, $\operatorname{CS}\left(G_{1}\right) \in \mathbb{R}^{c \times 1 \times r}, \operatorname{CS}\left(G_{2}\right) \in \mathbb{R}^{c \times r \times r}, \operatorname{CS}\left(G_{3}\right) \in$ $\mathbb{R}^{c \times r \times 1}$. We can then use the method proposed by (Pagh 2012) to compute a sequence of matrix multiplications.

- MTS We can rewrite the TT format as: $\operatorname{reshape}(\mathcal{T})=$ $\left(G_{1} \otimes G_{3}\right)$ reshape $\left(G_{2}\right)$. reshape $(\mathcal{T}) \in \mathbb{R}^{n_{1} n_{3} \times n_{2}}$, reshape $\left(G_{2}\right) \in \mathbb{R}^{r^{2} \times n_{2}}$. Algorithm 5 in Appendix A.2 shows the procedure steps.

Table 6: Computation and memory analysis for TT.

\begin{tabular}{|c|c|c|}
\hline Operator & Computation & Memory \\
\hline $\mathcal{T}$ & $n^{3} r^{2}$ & $O(n r)$ \\
\hline $\operatorname{CTS}(\mathcal{T})$ & $O\left(n r^{2}+c r^{2} \log c+c\right)$ & $O(n c)$ \\
\hline $\operatorname{MTS}(\mathcal{T})$ & $O\left(n r^{2}+m_{1} m_{2} \log \left(m_{1} m_{2}\right)\right)$ & $O\left(m_{1} m_{2}\right)$ \\
\hline
\end{tabular}

We present the error analysis for TT-form sketching in Appendix B.4 We show that if $c=O\left(r^{2}\right)$ and $m_{1} m_{2}=$ $O\left(r^{2}\right)$, the recovery error will be the same for MTS and CTS methods. This gives us an approximate $\mathrm{O}\left(r^{2}\right)$ times improvement using MTS if $\log r>n$, compared to using the CS method. 


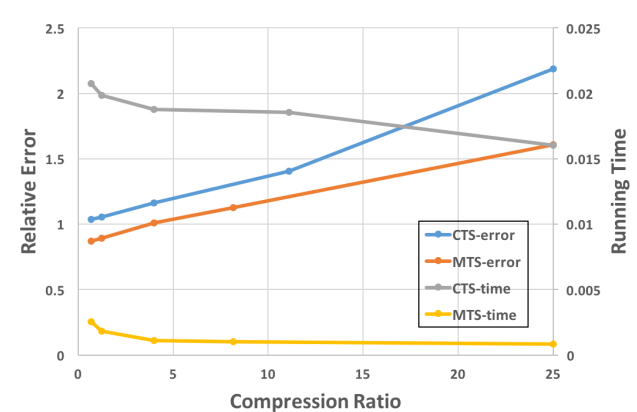

Figure 8: Kronecker product estimation for two $10 \times 10$ matrices.

\section{Experiments}

In this section, we present an empirical study of the multidimensional tensor sketch in matrix and tensor operations. The goals of this study are: a) establish that our method significantly compress the input without losing principle information from the data, b) demonstrate the advantages of MTS in compressing Kronecker product and tensor contraction operation in practice, compared to CTS, c) present potential application of MTS in deep learning tasks.

\subsection{Kronecker Product}

We compress Kronecker products using CTS and MTS based on Figure 5 and Figure 6. We compute the recovery relative error and compression time verse the compression ratio. Given a Kronecker product matrix $C \in \mathbb{R}^{a b \times d e}$, the $\operatorname{CTS}(C) \in \mathbb{R}^{a b \times c}$, the $\operatorname{MTS}(C) \in \mathbb{R}^{m_{1} \times m_{2}}$. The compression ratio for CTS and MTS are $\frac{d e}{c}$ and $\frac{a b d e}{m_{1} m_{2}}$. The relative error is calculated as $\frac{\|C-\hat{C}\|_{F}}{\|C\|_{F}}$. The result is obtained by independently running the sketching 5 times and choosing the median. All inputs are randomly generated from the normal distribution.

From Figure 8, we infer that the recovery error of CTS/MTS is proportional to the compression ratio, while the running time is inversely proportional to the compression ratio. MTS out performs CTS in terms of relative error and running time when they keep the same compression ratio.

\subsection{Covariance Matrix Estimation}

Given a matrix $A \in \mathbb{R}^{10 \times 10}$, all entries are sampled independently and uniformly from $[-1,1]$, except for rows two and nine which are positively correlated. In Figure 9 , the upper middle figure is the true value of $A A^{T}$, the upper right figure is an approximation of $A A^{T}$ using the algorithm in (Pagh, 2012) with a compression ratio of 2.5. Lower left is the true value of $A \otimes A^{T}$. By applying MS based on Figure 6(Algorithm 4 in Appendix A.1), we get the

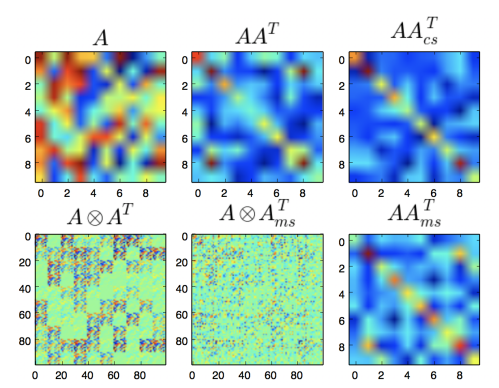

Figure 9: Covariance matrix estimation.

approximated $A \otimes A^{T}$ in the lower middle figure with a compression ratio of 6.25. Further, we estimate $A A^{T}$ using the fact that $\left(A A^{T}\right)_{i j}=\sum_{k=1}^{r}\left(A \otimes A^{T}\right)_{r(i-1)+k, n(k-1)+j}$, if $A \in \mathbb{R}^{n \times r}$. In both methods, we repeat the sketching 300 times and use the median as the final estimation. The covariance matrix estimation quality using MTS is better than the one using CS, and it uses a higher compression rate.

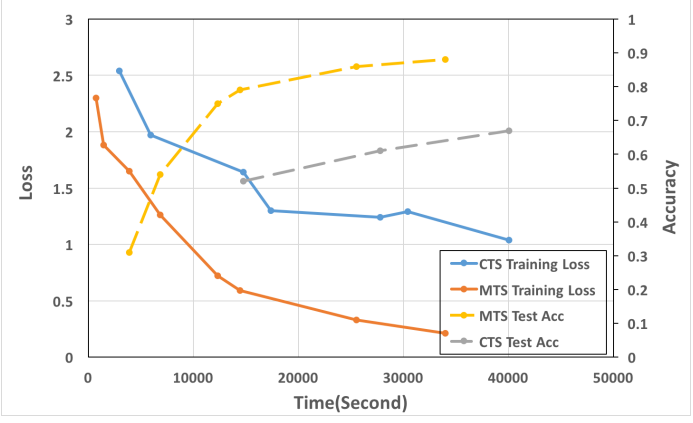

Figure 10: Training loss and test accuracy on CIFAR10 for different network structures.

\subsection{Tensor Regression Network}

To demonstrate the versatility of our method, we combine it with deep learning, by integrating it into a tensor regression network for object classification.

Kossaifi et al. (2017) propose the tensor regression layer(TRL) to express outputs through a low-rank multilinear mapping. It learns a Tucker-form tensor weight for the high-order activation tensor. Instead of reconstructing the full regression weight tensor using tensor contractions, we propose to sketch it using Equation 7 and 8 In our experiments, we use a ResNet18(He et al. 2016), from which the flattening and fully-connected layers are removed, and are replaced by our proposed sketched tensor regression layer. The network structure is illustrated in Figure 11 .

We compare CTS and MTS on the CIFAR10 dataset (Krizhevsky, 2009). We report the learning loss and the test accuracy in Figure 10 . Compared to CTS, 


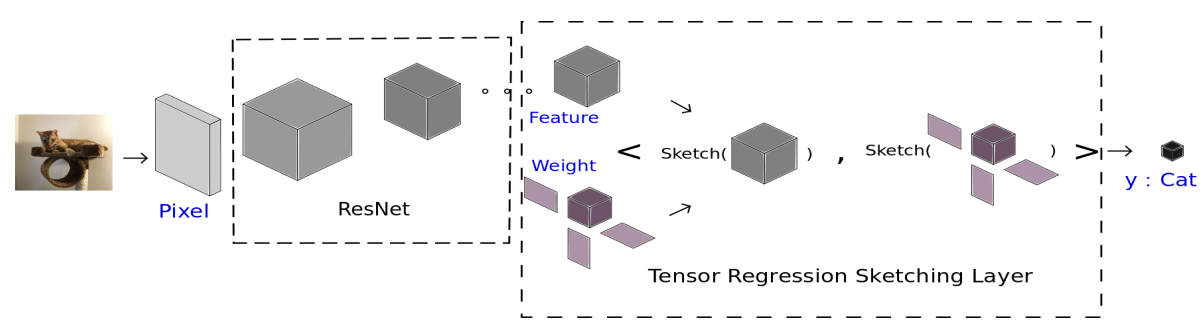

Figure 11: Tensor regression layer with sketching.

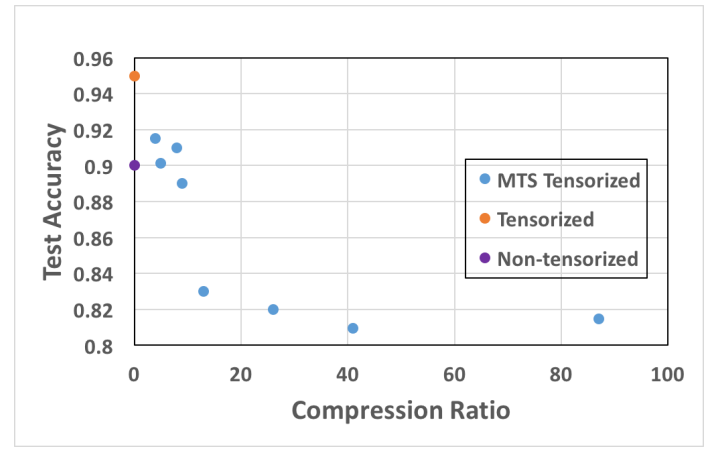

Figure 12: Test accuracy on CIFAR 10 for MTS tensorized network with different compression ratio(Compression ratio is respect to tensorized network. Compression ratio $=0$ means no compression added).

MTS converges faster. In Figure 12, MTS-tensorized network still outperforms the non-tensorized network when the compression ratio is not too big. Compared to the original tensor regression network, we use 8 times fewer parameters with only $4 \%$ accuracy loss(original TRL has $25 \mathrm{k}$ parameters, sketched TRL has 3k. Final test accuracy using original TRL/sketched TRL is $95 \% / 91 \%$ ).

\section{Related Work}

Given the importance of data approximation in the aspect of computation efficiency and privacy concerns, many methods have been developed to use fewer parameters in the estimation of the original data. As one might expect, data with a particular structure may require a specific approximation method. Truncated singular value decomposition (SVD) (Eckart \& Young, 1936) is an approximation method to represent the rotation and rescale of the data. However, this decomposition may not fit for specific data that has sparsity/non-negativity constraints. CX and CUR matrix decompositions are proposed to solve this problem (Mahoney \& Drineas, 2009, Caiafa \& Cichocki, 2010).

Since structure in the data may not be respected by mathematical operations on the data, sketching is more interpretable and has better informing intuition (Bringmann \& Panagiotou, 2017; Alon et al., 1999, Weinberger \& Saul,
2009). Min-hash (Broder, 1997) is a technique for estimating how similar two sets of data are. An extension of that is one-bit CP-hash (Christiani et al. 2018). To make use of parallel computing resources, 2-of-3 cuckoo hashing (Amossen \& Pagh 2011) is proposed based on (Pagh \& Rodler. 2001).

Count Sketch(CS) is first proposed by Charikar et al. (2002) to estimate the frequency of each element in a stream. Pagh (2012) propose a fast algorithm to compute count sketch of an outer product of two vectors using FFT properties. They prove that the CS of the outer product is equal to the convolutions between the CS of each input. Tensor sketch is proposed by Pham \& Pagh (2013) to approximate nonlinear kernel. It can approximate any polynomial kernel in $O(N(n+c \log c))$ time, while previous methods require $O(N n c)$ time for $N$ training samples in $n$-dimensional space and $c$ random feature maps. Wang et al. (2015) develop a novel approach for randomized computation of tensor contractions using tensor sketch, and apply it to fast tensor CP decomposition. However, all these sketching techniques are projecting tensors to vector space.

Besides above dimension reduction algorithms, efficient tensor contraction primitive is another focus in computation community. Jhurani \& Mullowney (2015) propose lowoverhead interface for multiple small matrix multiplications on NVIDIA GPUs. Shi et al. (2016) further present optimized computation primitives for single-index contractions involving all the possible configurations of second-order and third-order tensors.

\section{Conclusion}

In this paper, we propose a new sketching technique, called multi-dimensional tensor sketching (MTS). MTS is an unbiased estimator for tensors using sketching techniques along each mode. We present approximation algorithms for Kronecker product and tensor contraction. We improve both computational and memory requirement for tensor product and tensor contraction compared to previous sketching techniques, especially when tensors have high ranks. We apply the algorithms to synthetic and real data. In both cases, MTS performs efficient compression while preserving major information from the input. 


\section{Acknowledgements}

The authors would like to thank Jean Kossaifi for providing TRL code and Mikus Grasis for providing writing suggestions. Yang Shi is supported by Air Force Award FA955015-1-0221.

\section{References}

Alon, N., Matias, Y., and Szegedy, M. The space complexity of approximating the frequency moments. Journal of Computer and System Sciences, 58(1):137-147, February 1999.

Amossen, R. R. and Pagh, R. A new data layout for set intersection on gpus. In Proceedings of the 2011 IEEE International Parallel \& Distributed Processing Symposium, IPDPS '11, pp. 698-708, Washington, DC, USA, 2011. IEEE Computer Society. ISBN 978-0-76954385-7. doi: 10.1109/IPDPS.2011.71. URL https: //doi.org/10.1109/IPDPS.2011.71

Anandkumar, A., Ge, R., Hsu, D., Kakade, S. M., and Telgarsky, M. Tensor decompositions for learning latent variable models. The Journal of Machine Learning Research, 15(1):2773-2832, 2014.

Antol, S., Agrawal, A., Lu, J., Mitchell, M., Batra, D., Zitnick, C. L., and Parikh, D. VQA: Visual Question Answering. In International Conference on Computer Vision (ICCV), 2015.

Aza, Y., Fia, A., Karli, A. R., McSherr, F., and Sai, J. Spectral analysis of data. STOC, 2001.

Bringmann, K. and Panagiotou, K. Efficient sampling methods for discrete distributions. Algorithmica, 79(2): 484-508, Oct 2017. ISSN 1432-0541. doi: 10.1007/ s00453-016-0205-0. URL https://doi.org/10. $1007 / \mathrm{s} 00453-016-0205-0$

Broder, A. Z. On the resemblance and containment of documents. IEEE:Compression and Complexity of Sequences: Proceedings, Positano, Amalfitan Coast, Salerno, Italy, 10:21-29, 1997.

Caiafa, C. F. and Cichocki, A. Generalizing the column-row matrix decomposition to multi-way arrays. Linear Algebra and its Applications, 433:557-573, Sept 2010.

Charikar, M., Chen, K., and Farach-Colton, M. Finding frequent items in data streams. In Proceedings of ICALP'02, pp. 693-703, 2002.

Christiani, T., Pagh, R., and Sivertsen, J. Scalable and robust set similarity join. The annual IEEE International Conference on Data Engineering, 2018.
Demaine, E. D., López-Ortiz, A., and Munro, J. I. Frequency estimation of internet packet streams with limited space. In Proceedings of the 10th Annual European Symposium on Algorithms, ESA '02, pp. 348-360, London, UK, UK, 2002. Springer-Verlag. ISBN 3-540-441808. URL http://dl.acm.org/citation.cfm? id=647912.740658.

Eckart, C. and Young, G. The approximation of one matrix by another of lower rank. In Psychometrika. SpringerVerlag, 1936.

Fukui, A., Park, D. H., Yang, D., Rohrbach, A., Darrell, T., and Rohrbach, M. Multimodal compact bilinear pooling for visual question answering and visual grounding. EMNLP 2016, 2016.

Harshman, R. Foundations of the parafac procedure: Models and conditions for an explanatory multi-model factor analysis. UCLA Working Papers in Phonetics, 16: 1-84, 1970. URL http://citeseerx.ist.psu. edu/viewdoc/download?doi=10.1.1.144.

$5652 \& r e p=r e p 1 \& t y p e=p d f$

He, K., Zhang, X., Ren, S., and Sun, J. Deep residual learning for image recognition. In Proceedings of the IEEE Conference on Computer Vision and Pattern Recognition (CVPR)., 2016.

Jhurani, C. and Mullowney, P. A gemm interface and implementation on nvidia gpus for multiple small matrices. J. of Parallel and Distributed Computing, pp. 133-140, 2015.

Koren, Y., Bell, R., and Volinsky, C. Matrix factorization techniques for recommender systems. IEEE Computer Society, 2009.

Kossaifi, J., Lipton, Z. C., Khanna, A., Furlanello, T., and Anandkumar, A. Tensor regression networks. 2017. URL http://arxiv.org/abs/1707.08308.

Krizhevsky, A. Learning multiple layers of features from tiny images. Technical report, 2009.

Mahoney, M. W. and Drineas, P. Cur matrix decompositions for improved data analysis. Proceedings of the National Academy of Sciences, 106(3):697-702, 2009. ISSN 00278424. doi: 10.1073/pnas.0803205106.

Oseledets, I. V. Tensor-train decomposition. SIAM J. Sci. Comput, 33(5):2295-2317, 2011.

Pagh, R. Compressed matrix multiplication. ITCS, 2012.

Pagh, R. and Rodler, F. F. Cuckoo hashing. Lecture Notes in Computer Science, 2001. doi: 10.1007/3-540-44676-1_ 10. 
Pham, N. and Pagh, R. Fast and scalable polynomial kernels via explicit feature maps. KDD, 2013.

Shi, Y., Niranjan, U., Anandkumar, A., and Cecka, C. Tensor contractions with extended blas kernels on cpu and gpu. HiPC, 2016.

Shi, Y., Furlanello, T., Zha, S., and Anandkumar, A. Question type guidted attention in visual question answering. ECCV, 2018.

Springer, P., Su, T., and Bientinesi, P. Hptt: A highperformance tensor transposition c++ library. Proceedings of the 4th ACM SIGPLAN International Workshop on Libraries, Languages, and Compilers for Array Programming, 2017.

Teney, D., Anderson, P., He, X., and van den Hengel, A. Tips and tricks for visual question answering: Learnings from the 2017 challenge,. CVPR 2017 VQA Workshop, 2017. URL http://arxiv.org/abs/1708.02711

Tucker, L. R. Some mathematical notes on three-mode factor analysis. Psychometrika, 31(3):279-311, 1966. doi: 10.1007/BF02289464.

Wang, Y., Tung, H.-Y., Smola, A., and Anandkumar, A. Fast and guaranteed tensor decomposition via sketching. Proceedings of Advances in Neural Information Processing Systems (NIPS), 2015.

Weinberger, K. Q. and Saul, L. K. Distance metric learning for large margin nearest neighbor classification. Journal of Machine Learning Research, 10:207-244, 2009.

Yu, R., Zheng, S., Anandkumar, A., and Yue, Y. Long-term forecasting using tensor-train rnns. 


\section{A. List of some algorithms mentioned in the main paper}

\section{A.1. Kronecker Product Sketching}

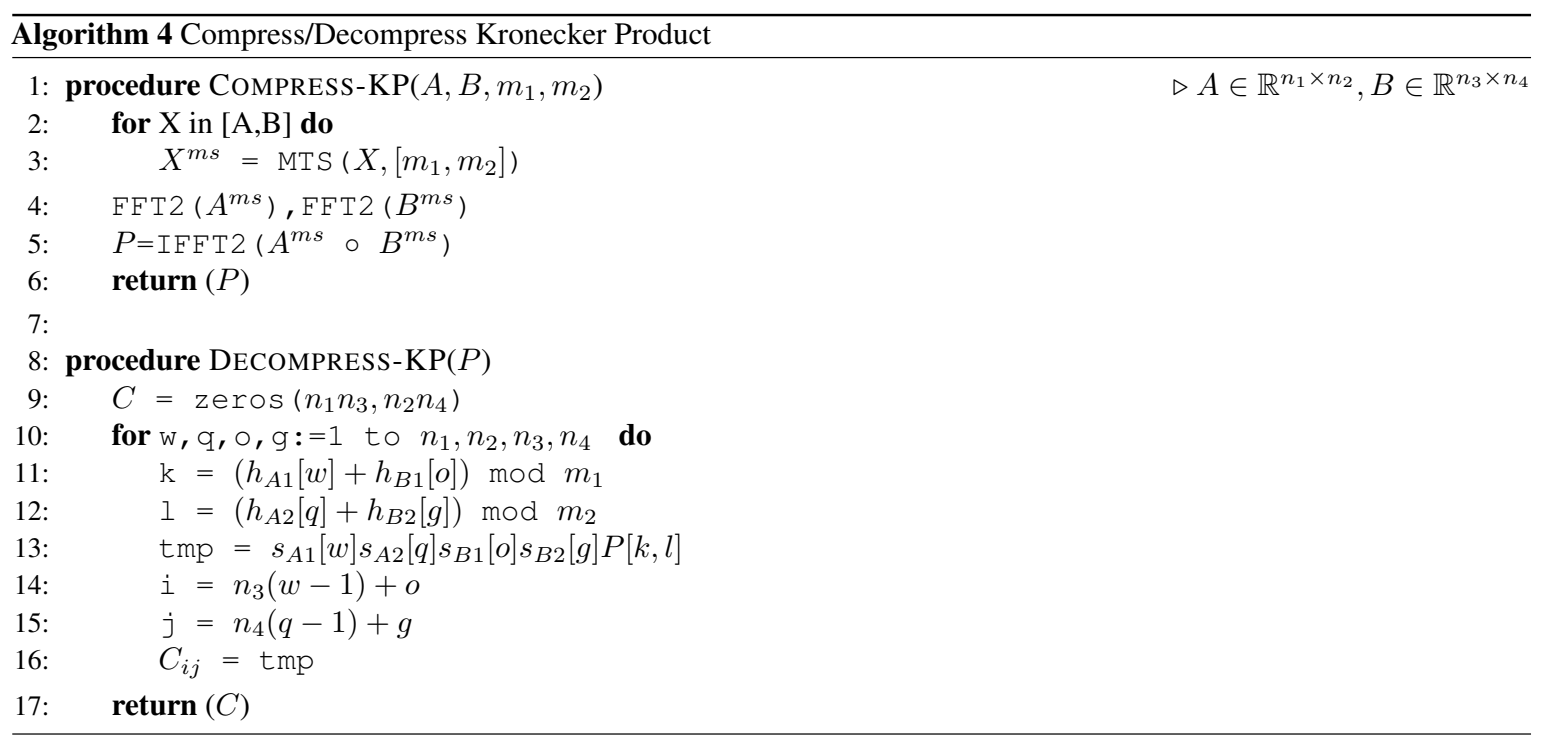

\section{A.2. Tensor-Train Sketching}

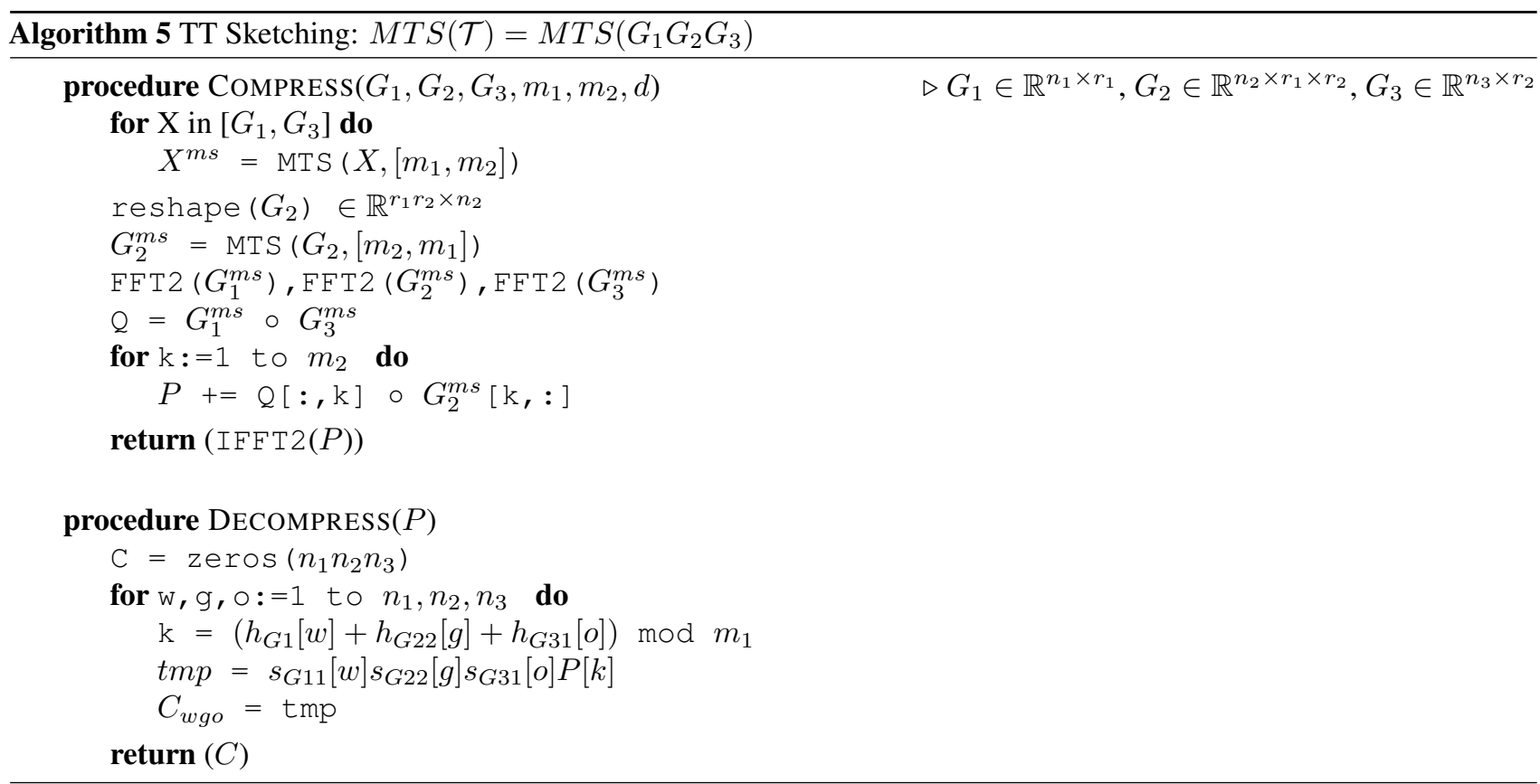

\section{B. Proofs of some technical theorems/lemmas}

\section{B.1. MS of the Kronecker product}

Lemma B.1. Given two matrices $A \in \mathbb{R}^{n \times n}, B \in \mathbb{R}^{n \times n}$,

$$
\begin{aligned}
\operatorname{MTS}(A \otimes B) & =\operatorname{MTS}(A) * \operatorname{MTS}(B) \\
& =\operatorname{IFFT2}(F F T 2(M T S(A)) \circ F F T 2(\operatorname{MTS}(B)))
\end{aligned}
$$


Proof of Lemma B.1 The Kronecker product defines $(A \otimes B)_{n_{3}(p-1)+h n_{4}(q-1)+g}=A_{p q} B_{h g}$. Thus:

$$
\begin{aligned}
& \sum_{p q h g}(A \otimes B)_{a b} s_{1}(p) s_{2}(q) s_{3}(h) s_{4}(g) w^{t_{1} h_{a}+t_{2} h_{b}} \\
& =\sum_{p q h g} A_{p q} B_{h g} s_{1}(p) s_{2}(q) s_{3}(h) s_{4}(g) w^{t_{1} h_{a}+t_{2} h_{b}} \\
& =\sum_{p q} A_{p q} s_{1}(p) s_{2}(q) w^{t_{1} h_{1}(p)+t_{2} h_{2}(q)} \sum_{h g} B_{h g} s_{3}(h) s_{4}(g) w^{t_{1} h_{3}(h)+t_{2} h_{4}(g)} \\
& =\operatorname{FFT} 2(M T S(A)) \circ F F T 2(M T S(B))
\end{aligned}
$$

where $a=n_{3}(p-1)+h, b=n_{4}(q-1)+g, h_{a}=h_{1}(p)+h_{3}(h), h_{b}=h_{2}(q)+h_{4}(g)$.

Assign $i=n_{3}(p-1)+h, j=n_{4}(q-1)+g, s_{5}(i)=s_{1}(p) s_{3}(h), s_{6}(j)=s_{1}(q) s_{3}(g), h_{5}(i)=h_{1}(p)+h_{3}(h)$ and $h_{6}(i)=h_{2}(q)+h_{4}(g)$, we have

$$
\begin{aligned}
& \sum_{p q h g}(A \otimes B)_{a b} s_{1}(p) s_{2}(q) s_{3}(h) s_{4}(g) w^{t_{1} h_{a}+t_{2} h_{b}} \\
& =\sum_{i j}(A \otimes B)_{i j} s_{5}(i) s_{6}(j) w^{t_{1} h_{5}(i)+t_{2} h_{6}(j)} \\
& =F F T 2(M T S(A \otimes B)) \\
& =F F T 2(M T S(A)) \circ F F T 2(M S(B))
\end{aligned}
$$

Consequently, we have $M T S(A \otimes B)=\operatorname{IFFT2}(F F T 2(M T S(A)) \circ F F T 2(M T S(B)))$. The recovery map is

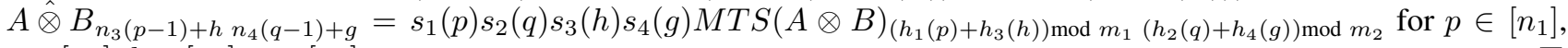
$q \in\left[n_{2}\right], h \in\left[n_{3}\right], g \in\left[n_{4}\right]$.

\section{B.2. Analysis of CS and MTS approximation error}

Theorem B.2 ((Charikar et al. 2002)). Given a vector $u \in \mathbb{R}^{n}, \mathrm{CS}$ hashing functions s and $\mathrm{h}$ with sketching dimension c, for any $i^{*}$, the recovery function $\hat{u}\left(i^{*}\right)=s\left(i^{*}\right) C S(u)\left(h\left(i^{*}\right)\right)$ computes an unbiased estimator for $u\left(i^{*}\right)$ with variance bounded by $\|u\|_{2}^{2} / c$.

Proof of Theorem B.2 For $i \in\{1,2, \cdots n\}$, let $K_{i}$ be the indicator variable for the event $h(i)=h\left(i^{*}\right)$. We can write $\hat{u}\left(i^{*}\right)$ as

$$
\hat{u}\left(i^{*}\right)=s\left(i^{*}\right) \sum_{i} K_{i} s(i) u(i)
$$

Observe that $K_{i}=1$, if $i=i^{*}, \mathbf{E}\left(s\left(i^{*}\right) s(i)\right)=0$, for all $i \neq i^{*}$, and $\mathbf{E}\left(s\left(i^{*}\right)^{2}\right)=1$, we have

$$
\begin{aligned}
\mathbf{E}\left(\hat{u}\left(i^{*}\right)\right) & =\mathbf{E}\left(s\left(i^{*}\right) K_{i^{*}} s\left(i^{*}\right) u\left(i^{*}\right)\right)+\mathbf{E}\left(s\left(i^{*}\right) \sum_{i \neq i^{*}} K_{i} s(i) u(i)\right) \\
& =u(i)
\end{aligned}
$$

To bound the variance, we rewrite the recovery function as

$$
\hat{u}\left(i^{*}\right)=s\left(i^{*}\right) K_{i^{*}} s\left(i^{*}\right) u\left(i^{*}\right)+s\left(i^{*}\right) \sum_{i \neq i^{*}} K_{i} s(i) u(i)
$$

To simplify notation, we assign $\mathrm{X}$ as the first term, $\mathrm{Y}$ as the second term. $\operatorname{Var}(X)=0$, and $\operatorname{COV}(X, Y)=0$ since $s(i)$ for $i \in\{1,2, \cdots n\}$ are 2 -wise independent. Thus,

$$
\operatorname{Var}(X+Y)=\sum_{i \neq i^{*}} \operatorname{Var}\left(K_{i} s\left(i^{*}\right) s(i) u(i)\right)
$$


$\mathbf{E}\left(K_{i} s\left(i^{*}\right) s(i) u(i)\right)=0$ for $i \neq i^{*}$. Consequently,

$$
\operatorname{Var}\left(K_{i} s\left(i^{*}\right) s(i) u(i)\right)=\mathbf{E}\left(\left(K_{i} s\left(i^{*}\right) s(i) u(i)\right)^{2}\right)=\mathbf{E}\left(K_{i}^{2}\right) u(i)^{2}=u(i)^{2} / c
$$

The last equality uses that $\mathbf{E}\left(K_{i}^{2}\right)=\mathbf{E}\left(K_{i}\right)=1 / c$, for all $i \neq i^{*}$. Summing over all terms, we have $\operatorname{Var}\left(\hat{u}\left(i^{*}\right)\right) \leq$ $\|u\|_{2}^{2} / c$.

Proof of Theorem 2.1. For $i \in\left\{1,2, \cdots n_{1}\right\}, j \in\left\{1,2, \cdots n_{2}\right\}$, let $K_{i j}$ be the indicator variable for the event $h_{1}(i)=$ $h_{1}\left(i^{*}\right)$ and $h_{2}(j)=h_{2}\left(j^{*}\right)$. We can write $\hat{U}_{i^{*} j^{*}}$ as

$$
\hat{U}_{i^{*} j^{*}}=s_{1}\left(i^{*}\right) s_{2}\left(j^{*}\right) \sum_{i j} K_{i j} s_{1}(i) s_{2}(j) U_{i j}
$$

Observe that $K_{i j}=1$, if $i=i^{*}, j=j^{*}$. $\mathbf{E}\left(s_{1}\left(i^{*}\right) s_{1}(i)\right)=0, \mathbf{E}\left(s_{2}\left(j^{*}\right) s_{2}(j)\right)=0$, for all $i \neq i^{*}, j \neq j^{*}$, and $\mathbf{E}\left(s_{1}\left(i^{*}\right)^{2}\right)=1, \mathbf{E}\left(s_{2}\left(j^{*}\right)^{2}\right)=1$, we have

$$
\begin{aligned}
\mathbf{E}\left(\hat{U}_{i^{*} j^{*}}\right) & =\mathbf{E}\left(s_{1}^{2}\left(i^{*}\right) s_{2}^{2}\left(j^{*}\right) K_{i^{*} j^{*}} U_{i^{*} j^{*}}+\mathbf{E}\left(s_{1}\left(i^{*}\right) s_{2}\left(j^{*}\right) \sum_{i \neq i^{*}, j \neq j^{*}} K_{i j} s_{1}(i) s_{2}(j) U_{i j}\right)\right. \\
& =U_{i^{*} j^{*}}
\end{aligned}
$$

To bound the variance, we rewrite the recovery function as

$$
\hat{U}_{i^{*} j^{*}}=s_{1}^{2}\left(i^{*}\right) s_{2}^{2}\left(j^{*}\right) K_{i^{*} j^{*}} U_{i^{*} j^{*}}+s_{1}\left(i^{*}\right) s_{2}\left(j^{*}\right) \sum_{i \neq i^{*}, j \neq j^{*}} K_{i j} s_{1}(i) s_{2}(j) U_{i j}
$$

To simplify notation, we assign $\mathrm{X}$ as the first term, $\mathrm{Y}$ as the second term. $\operatorname{Var}(X)=0$, and $C O V(X, Y)=0$ since $s_{1}(i)$ and $s_{2}(j)$ for $i \in\left\{1,2, \cdots n_{1}\right\}, j \in\left\{1,2, \cdots n_{2}\right\}$ are both 2-wise independent. Thus,

$$
\operatorname{Var}(X+Y)=\sum_{i \neq i^{*}, j \neq j^{*}} \operatorname{Var}\left(K_{i j} s_{1}\left(i^{*}\right) s_{2}\left(j^{*}\right) s_{1}(i) s_{2}(j) U_{i j}\right)
$$

$\mathbf{E}\left(K_{i j} s_{1}\left(i^{*}\right) s_{2}\left(j^{*}\right) s_{1}(i) s_{2}(j) U_{i j}\right)=0$ for $i \neq i^{*}, j \neq j^{*}$. Consequently,

$$
\operatorname{Var}\left(K_{i j} s_{1}(i) s_{2}(j) U_{i j}\right)=\mathbf{E}\left(\left(K_{i j} s_{1}\left(i^{*}\right) s_{2}\left(j^{*}\right) s_{1}(i) s_{2}(j) U_{i j}\right)^{2}\right)=\mathbf{E}\left(K_{i j}^{2}\right) U_{i j}^{2}=U_{i j}^{2} /\left(m_{1} m_{2}\right)
$$

The last equality uses that $\mathbf{E}\left(K_{i j}^{2}\right)=\mathbf{E}\left(K_{i j}\right)=1 /\left(m_{1} m_{2}\right)$, for all $i \neq i^{*}, j \neq j^{*}$. Summing over all terms, we have $\operatorname{Var}\left(\hat{U}_{i^{*} j^{*}}\right) \leq\|U\|_{F}^{2} /\left(m_{1} m_{2}\right)$.

\section{B.3. Analysis of Tucker-form tensor approximation error}

Proof of Theorem 3.1. We first prove the unbiasedness.

$$
\begin{aligned}
\mathbf{E}\left(\hat{\mathcal{T}}_{i j k}\right) & =\sum_{a=1}^{r} \sum_{b=1}^{r} \sum_{c=1}^{r} \mathcal{G}_{a b c} \mathbf{E}\left(\hat{U}_{i a} \hat{V}_{j b} \hat{W}_{k c}\right) \\
& =\sum_{a=1}^{r} \sum_{b=1}^{r} \sum_{c=1}^{r} \mathcal{G}_{a b c} \mathbf{E}\left(\hat{U}_{i a}\right) \mathbf{E}\left(\hat{V}_{j b}\right) \mathbf{E}\left(\hat{W}_{k c}\right) \\
& =\sum_{a=1}^{r} \sum_{b=1}^{r} \sum_{c=1}^{r} \mathcal{G}_{a b c} U_{i a} V_{j b} W_{k c} \\
& =\mathcal{T}_{i j k}
\end{aligned}
$$


The second step uses the property that the elements are independent. The third step is obtained using Theorem B.2

$$
\begin{aligned}
\operatorname{Var}\left(\hat{\mathcal{T}}_{i j k}\right) & =\sum_{a=1}^{r} \sum_{b=1}^{r} \sum_{c=1}^{r} \mathcal{G}_{a b c} \operatorname{Var}\left(\hat{U}_{i a} \hat{V}_{j b} \hat{W}_{k c}\right) \\
& =\sum_{a=1}^{r} \sum_{b=1}^{r} \sum_{c=1}^{r} \mathcal{G}_{a b c}\left(\operatorname{Var}\left(\hat{U}_{i a}\right) \operatorname{Var}\left(\hat{V}_{j b}\right) \operatorname{Var}\left(\hat{W}_{k c}\right)+\mathbf{E}^{2}\left(\hat{U}_{i a}\right) \operatorname{Var}\left(\hat{V}_{j b}\right) \operatorname{Var}\left(\hat{W}_{k c}\right)\right. \\
& +\mathbf{E}^{2}\left(\hat{V}_{j b}\right) \operatorname{Var}\left(\hat{U}_{i a}\right) \operatorname{Var}\left(\hat{W}_{k c}\right)+\mathbf{E}^{2}\left(\hat{W}_{k c}\right) \operatorname{Var}\left(\hat{U}_{i a}\right) \operatorname{Var}\left(\hat{V}_{j b}\right) \\
& +\mathbf{E}^{2}\left(\hat{U}_{i a}\right) \mathbf{E}^{2}\left(\hat{V}_{j b}\right) \operatorname{Var}\left(\hat{W}_{k c}\right)+\mathbf{E}^{2}\left(\hat{V}_{j b}\right) \mathbf{E}^{2}\left(\hat{W}_{k c}\right) \operatorname{Var}\left(\hat{U}_{i a}\right) \\
& \left.+\mathbf{E}^{2}\left(\hat{U}_{i a}\right) \mathbf{E}^{2}\left(\hat{W}_{k c}\right) \operatorname{Var}\left(\hat{V}_{j b}\right)\right) \\
& \leq \sum_{a=1}^{r} \sum_{b=1}^{r} \sum_{c=1}^{r} \mathcal{G}_{a b c}\left(\frac{\left\|U_{a}\right\|_{2}^{2}\left\|V_{b}\right\|_{2}^{2}\left\|W_{c}\right\|_{2}^{2}}{c^{3}}+\frac{U_{i a}^{2}\left\|V_{b}\right\|_{2}^{2}\left\|W_{c}\right\|_{2}^{2}}{c^{2}}\right. \\
& \left.+\frac{V_{j b}^{2}\left\|U_{a}\right\|_{2}^{2}\left\|W_{c}\right\|_{2}^{2}}{c^{2}}+\frac{W_{k c}^{2}\left\|U_{a}\right\|_{2}^{2}\left\|V_{b}\right\|_{2}^{2}}{c^{2}}+\frac{U_{i a}^{2} V_{j b}^{2}\left\|W_{c}\right\|_{2}^{2}}{c}+\frac{V_{j b}^{2} W_{k c}^{2}\left\|U_{a}\right\|_{2}^{2}}{c}+\frac{U_{i a}^{2} W_{k c}^{2}\left\|V_{b}\right\|_{2}^{2}}{c}\right) \\
& \leq r^{3} g\left\|U_{a}\right\|_{2}^{2}\left\|V_{b}\right\|_{2}^{2}\left\|W_{c}\right\|_{2}^{2}\left(\frac{1}{c^{3}}+\frac{3}{c^{2}}+\frac{3}{c}\right) \\
& \leq 7 r^{3} g\left\|U_{a}\right\|_{2}^{2}\left\|V_{b}\right\|_{2}^{2}\left\|W_{c}\right\|_{2}^{2} \frac{1}{c}
\end{aligned}
$$

Computation complexity analysis $\operatorname{CTS}(\mathcal{T})$ can be computed using $O\left(r^{3}(n+c \log c+c)\right)$ operators: Inside $r^{3}$ summations, it takes $O(n)$ to do the count sketch for each column of $U, V$, and $W, O(c \log c)$ to do FFT/IFFT, and $O(c)$ to do elementwise product between the FFT results. The memory complexity is $O\left(c r+r^{3}\right): O(c r)$ to store the count sketch results of $U, V, W, O\left(r^{3}\right)$ to store $\mathcal{G}$.

\section{Proof of Theorem 3.2}

$$
\begin{aligned}
\mathbf{E}\left(\hat{\mathcal{T}}_{i j k}\right) & =\mathbf{E}\left(\sum_{l=1}^{r^{3}}(\hat{U} \otimes \hat{V} \otimes \hat{W})_{i j k l} \operatorname{vec}(\hat{\mathcal{G}})_{l}\right) \\
& =\sum_{l=1}^{r^{3}} \mathbf{E}(\hat{U} \otimes \hat{V} \otimes \hat{W})_{i j k l} \mathbf{E}\left(\operatorname{vec}(\hat{\mathcal{G}})_{l}\right) \\
& =\sum_{l=1}^{r^{3}}(U \otimes V \otimes W)_{i j k l} \operatorname{vec}(\mathcal{G})_{l} \\
& =\mathcal{T}_{i j k}
\end{aligned}
$$

This is an unbiased estimator because both MS and CS are unbiased. For the variance, we have:

$$
\begin{aligned}
\operatorname{Var}\left(\hat{\mathcal{T}}_{i j k}\right) & =\operatorname{Var}\left(\sum_{l=1}^{r^{3}}(\hat{U} \otimes \hat{V} \otimes \hat{W})_{i j k l} \operatorname{vec}(\hat{\mathcal{G}})_{l}\right) \\
& =\sum_{l=1}^{r^{3}} \operatorname{Var}(\hat{U} \otimes \hat{V} \otimes \hat{W})_{i j k l} \operatorname{vec}(\hat{\mathcal{G}})_{l}
\end{aligned}
$$

To simplify the notation, we set $X=\hat{U} \otimes \hat{V} \otimes \hat{W}, Y=\operatorname{vec}(\hat{\mathcal{G}})$.

From kronecker product definition, we know $\exists a, b, c, d, e, f, X_{t l}=\hat{U}_{a b} \hat{V}_{c d} \hat{W}_{e f}$, for $\forall t, l$. Thus, $\operatorname{Var}\left(X_{t l}\right)=$ 
$\operatorname{Var}\left(\hat{U}_{a b} \hat{V}_{c d} \hat{W}_{e f}\right)$.

$$
\begin{aligned}
\operatorname{Var}\left(\hat{U}_{a b} \hat{V}_{c d} \hat{W}_{e f}\right) & =\operatorname{Var}\left(\hat{U}_{a b}\right) \operatorname{Var}\left(\hat{V}_{c d}\right) \operatorname{Var}\left(\hat{W}_{e f}\right)+\mathbf{E}^{2}\left(\hat{U}_{a b}\right) \operatorname{Var}\left(\hat{V}_{c d}\right) \operatorname{Var}\left(\hat{W}_{e f}\right) \\
& +\mathbf{E}^{2}\left(\hat{V}_{c d}\right) \operatorname{Var}\left(\hat{U}_{a b}\right) \operatorname{Var}\left(\hat{W}_{e f}\right)+\mathbf{E}^{2}\left(\hat{W}_{e f}\right) \operatorname{Var}\left(\hat{U}_{a b}\right) \operatorname{Var}\left(\hat{V}_{c d}\right) \\
& +\mathbf{E}^{2}\left(\hat{U}_{a b}\right) \mathbf{E}^{2}\left(\hat{V}_{c d}\right) \operatorname{Var}\left(\hat{W}_{e f}\right)+\mathbf{E}^{2}\left(\hat{V}_{c d}\right) \mathbf{E}^{2}\left(\hat{W}_{e f}\right) \operatorname{Var}\left(\hat{U}_{a b}\right) \\
& +\mathbf{E}^{2}\left(\hat{U}_{a b}\right) \mathbf{E}^{2}\left(\hat{W}_{e f}\right) \operatorname{Var}\left(\hat{V}_{c d}\right) \\
& =\frac{\|U\|_{F}^{2}\|V\|_{F}^{2}\|W\|_{F}^{2}}{\left(m_{1} m_{2}\right)^{3}}+\frac{U_{a b}^{2}\|V\|_{F}^{2}\|W\|_{F}^{2}}{\left(m_{1} m_{2}\right)^{2}} \\
& +\frac{V_{c d}^{2}\|U\|_{F}^{2}\|W\|_{F}^{2}}{\left(m_{1} m_{2}\right)^{2}}+\frac{W_{e f}^{2}\|U\|_{2}^{2}\|V\|_{F}^{2}}{\left(m_{1} m_{2}\right)^{2}} \\
& +\frac{U_{a b}^{2} V_{c d}^{2}\|W\|_{F}^{2}}{m_{1} m_{2}}+\frac{V_{c d}^{2} W_{e f}^{2}\|U\|_{F}^{2}}{m_{1} m_{2}}+\frac{U_{a b}^{2} W_{e f}^{2}\|V\|_{F}^{2}}{m_{1} m_{2}} \\
& \leq 7\|U\|_{F}^{2}\|V\|_{F}^{2}\|W\|_{F}^{2} \frac{1}{m_{1} m_{2}}
\end{aligned}
$$

We know $\operatorname{Var}\left(Y_{l}\right)=\frac{\|\mathcal{G}\|_{F}^{2}}{m_{2}}, \mathbf{E}\left(X_{t l}\right)=U_{a b} V_{c d} W_{e f}, \mathbf{E}\left(Y_{l}\right)=\mathcal{G}_{l}$. Thus,

$$
\begin{aligned}
\operatorname{Var}\left(\mathcal{T}_{i j k}\right) & =\operatorname{Var}\left(\sum_{l}^{r^{3}} X_{t l} Y_{l}\right) \\
& =\sum_{l}^{r^{3}} \operatorname{Var}\left(X_{t l}\right) \operatorname{Var}\left(Y_{l}\right)+\mathbf{E}^{2}\left(X_{t l}\right) \operatorname{Var}\left(Y_{l}\right)+\mathbf{E}^{2}\left(Y_{l}\right) \operatorname{Var}\left(X_{t l}\right) \\
& \leq \sum_{l}^{r^{3}} 7\|U\|_{F}^{2}\|V\|_{F}^{2}\|W\|_{F}^{2}\|\mathcal{G}\|_{F}^{2} \frac{1}{m_{1} m_{2}^{2}}+\mathbf{E}^{2}\left(X_{t l}\right) \frac{\|\mathcal{G}\|_{F}^{2}}{m_{2}}+\mathbf{E}^{2}\left(Y_{l}\right) 7\|U\|_{F}^{2}\|V\|_{F}^{2}\|W\|_{F}^{2} \frac{1}{m_{1} m_{2}} \\
& \leq \sum_{l}^{r^{3}}\|U\|_{F}^{2}\|V\|_{F}^{2}\|W\|_{F}^{2}\|\mathcal{G}\|_{F}^{2}\left(\frac{7}{m_{1} m_{2}^{2}}+\frac{1}{m_{2}}+\frac{7}{m_{1} m_{2}}\right) \\
& \leq c_{0} r^{3}\|U\|_{F}^{2}\|V\|_{F}^{2}\|W\|_{F}^{2}\|\mathcal{G}\|_{F}^{2} \frac{1}{m_{1} m_{2}}
\end{aligned}
$$

$c_{0}$ is a constant number such that $c_{0} \geq 7+m_{1}+\frac{7}{m_{2}}$.

From Chebychev's inequality, if we run this sketch $d$ times, where $d=\Omega(\log (1 / \delta))$, we can get the desired error bond with probability at least $1-\delta$.

Computation complexity analysis Overall, this sketch requires $O\left(n r+r^{3}+m_{1} m_{2} \log \left(m_{1} m_{2}\right)+m_{1} m_{2}\right)$ operations if $m_{1}, m_{2}$ are the sketching lengths for the two modes for $U, V, W$ : it takes $O\left(n r+r^{3}\right)$ to do the MTS for $U, V, W$ and CS for vec(G). FFT2 requires $O\left(m_{1} m_{2} \log \left(m_{1} m_{2}\right)\right)$. We also need $m_{1} m_{2}$ operations to complete the element-wise product computation. The memory complexity is $O\left(m_{1} m_{2}\right)$.

\section{B.4. Analysis of Tensor-Train form tensor approximation error}

Theorem B.3. Suppose $\hat{\mathcal{T}} \in \mathbb{R}^{n \times n \times n}$ where $\hat{\mathcal{T}}=\hat{G}_{1} \hat{G}_{2} \hat{G}_{3}, \hat{G}_{1} \in \mathbb{R}^{n \times r}, \hat{G}_{2} \in \mathbb{R}^{n \times r \times r}, \hat{G}_{3} \in \mathbb{R}^{n \times r}$. $\hat{\mathcal{T}}$ is the recovered tensor for $\mathcal{T}=G_{1} G_{2} G_{3}$ after applying count sketch on $G_{1}, G_{2}$ and $G_{3}$ with sketching dimension c. Suppose the estimation takes $d$ independent sketches of $G_{1}, G_{2}$ and $G_{3}$ and then report the median of the d estimates. If $d=$ $\Omega\left(\log \left(n r^{2} / \delta\right)\right), \max \left(\left\|G_{1}\left[:, l_{1}\right]\right\|_{2}\right)=g_{1}, \max \left(\left\|G_{2}\left[:, l_{1}, l_{2}\right]\right\|_{2}\right)=g_{2}, \max \left(\left\|G_{3}\left[:, l_{2}\right]\right\|_{2}\right)=g_{3}$ where $l_{1} \in[r], l_{2} \in[r]$, and $c=\Omega\left(\frac{7 r^{2} g_{1}^{2} g_{2}^{2} g_{3}^{2}}{\epsilon^{2}}\right)$, then with probability $\geq 1-\delta$ there is $\left|\hat{\mathcal{T}}_{i j k}-T_{i j k}\right| \leq \epsilon$. 
Proof of Theorem B.3.

$$
\begin{aligned}
\operatorname{Var}\left(\hat{T}_{i j k}\right) & =\operatorname{Var}\left(\hat{G}_{1}[i] \hat{G}_{2}[j] \hat{G}_{3}[k]\right) \\
& =\operatorname{Var}\left(\sum_{l_{1}}^{r} \sum_{l_{2}}^{r} G_{1}\left[i, l_{1}\right] G_{2}\left[j, l_{1}, l_{2}\right] G_{3}\left[k, l_{2}\right]\right) \\
& =\sum_{l_{1}}^{r} \sum_{l_{2}}^{r} \operatorname{Var}\left(\hat{G}_{1}\left[i, l_{1}\right]\right) \operatorname{Var}\left(\hat{G}_{2}\left[j, l_{1}, l_{2}\right]\right) \operatorname{Var}\left(\hat{G}_{3}\left[k, l_{2}\right]\right)+\mathbf{E}^{2}\left(\hat{G}_{1}\left[i, l_{1}\right]\right) \operatorname{Var}\left(\hat{G}_{2}\left[j, l_{1}, l_{2}\right]\right) \operatorname{Var}\left(\hat{G}_{3}\left[k, l_{2}\right]\right) \\
& +\mathbf{E}^{2}\left(\hat{G}_{2}\left[j, l_{1}, l_{2}\right]\right) \operatorname{Var}\left(\hat{G}_{1}\left[i, l_{1}\right]\right) \operatorname{Var}\left(\hat{G}_{3}\left[k, l_{2}\right]\right)+\mathbf{E}^{2}\left(\hat{G}_{3}\left[k, l_{2}\right]\right) \operatorname{Var}\left(\hat{G}_{1}\left[i, l_{1}\right]\right) \operatorname{Var}\left(\hat{G}_{2}\left[j, l_{1}, l_{2}\right]\right) \\
& +\mathbf{E}^{2}\left(\hat{G}_{1}\left[i, l_{1}\right]\right) \mathbf{E}^{2}\left(\hat{G}_{2}\left[j, l_{1}, l_{2}\right]\right) \operatorname{Var}\left(\hat{G}_{3}\left[k, l_{2}\right]\right)+\mathbf{E}^{2}\left(\hat{G}_{2}\left[j, l_{1}, l_{2}\right]\right) \mathbf{E}^{2}\left(\hat{G}_{3}\left[k, l_{2}\right]\right) \operatorname{Var}\left(\hat{G}_{1}\left[i, l_{1}\right]\right) \\
& +\mathbf{E}^{2}\left(\hat{G}_{1}\left[i, l_{1}\right]\right) \mathbf{E}^{2}\left(\hat{G}_{3}\left[k, l_{2}\right]\right) \operatorname{Var}\left(\hat{G}_{2}\left[j, l_{1}, l_{2}\right]\right) \\
& \leq \sum_{l_{1}}^{r} \sum_{l_{2}}^{r} \frac{\left\|G_{1}\left[:, l_{1}\right]\right\|_{2}^{2}\left\|G_{2}\left[:, l_{1}, l_{2}\right]\right\|_{2}^{2}\left\|G_{3}\left[:, l_{2}\right]\right\|_{2}^{2}}{c^{3}}+\frac{G_{1}^{2}\left[i, l_{1}\right]\left\|G_{2}\left[:, l_{1}, l_{2}\right]\right\|_{2}^{2}\left\|G_{3}\left[:, l_{2}\right]\right\|_{2}^{2}}{c^{2}} \\
& +\frac{G_{2}^{2}\left[j, l_{1}, l_{2}\right]\left\|G_{1}\left[:, l_{1}\right]\right\|_{2}^{2}\left\|G_{3}\left[:, l_{2}\right]\right\|_{2}^{2}}{c^{2}}+\frac{G_{3}^{2}\left[k, l_{2}\right]\left\|G_{2}\left[:, l_{1}, l_{2}\right]\right\|_{2}^{2}\left\|G_{1}\left[:, l_{1}\right]\right\|_{2}^{2}}{c^{2}} \\
& +\frac{G_{1}^{2}\left[i, l_{1}\right] G_{2}^{2}\left[j, l_{1}, l_{2}\right]\left\|G_{3}\left[:, l_{2}\right]\right\|_{2}^{2}}{c}+\frac{G_{2}^{2}\left[j, l_{1}, l_{2}\right] G_{3}^{2}\left[k, l_{2}\right]\left\|G_{1}\left[:, l_{1}\right]\right\|_{2}^{2}}{c}+\frac{G_{1}^{2}\left[i, l_{1}\right] G_{3}^{2}\left[k, l_{2}\right]\left\|G_{2}\left[:, l_{1}, l_{2}\right]\right\|_{2}^{2}}{c} \\
& \leq r^{2} G_{1 a}^{2} G_{2 b}^{2} G_{3 c}^{2}\left(\frac{1}{c^{3}}+\frac{3}{c^{2}}+\frac{3}{c}\right) \\
& \leq 7 r^{2} G_{1 a}^{2} G_{2 b}^{2} G_{3 c}^{2} \frac{1}{c}
\end{aligned}
$$

when we assume $G_{1 a}=\max \left(G_{1}\left[:, l_{1}\right]\right), G_{2 b}=\max \left(G_{2}\left[:, l_{1}, l_{2}\right]\right), G_{3 c}=\max \left(G_{3}\left[:, l_{2}\right]\right)$ for $l_{1} \in\left[r_{1}\right], l_{2} \in\left[r_{2}\right]$.

Theorem B.4. Suppose $\hat{\mathcal{T}} \in \mathbb{R}^{n \times n \times n}$ where $\hat{\mathcal{T}}=\hat{G}_{1} \hat{G}_{2} \hat{G}_{3}, \hat{G}_{1} \in \mathbb{R}^{n \times r}, \hat{G}_{2} \in \mathbb{R}^{n \times r \times r}, \hat{G}_{3} \in \mathbb{R}^{n \times r}$. $\hat{\mathcal{T}}$ is the recovered tensor for $\mathcal{T}=G_{1} G_{2} G_{3}$ after applying multi-dimensional tensor sketch on $G_{1}$, reshape $\left(G_{2}\right)$ and $G_{3}$ with sketching dimension $m_{1}, m_{2}$. Suppose the estimation takes d independent sketches of $G_{1}$, reshape $\left(G_{2}\right)$ and $G_{3}$ and then report the median of the d estimates. If $d=\Omega\left(\log \left(n r^{2} / \delta\right)\right), \max \left(\left\|G_{1}\right\|_{F}\right)=g_{1}, \max \left(\mid G_{2} \|_{F}\right)=g_{2}, \max \left(\left\|G_{3}\right\|_{F}\right)=g_{3}$ and $m_{1} m_{2}=\Omega\left(\frac{7 r^{2} g_{1}^{2} g_{2}^{2} g_{3}^{2}}{\epsilon^{2}}\right)$, then with probability $\geq 1-\delta$ there is $\left|\hat{\mathcal{T}}_{i j k}-T_{i j k}\right| \leq \epsilon$.

Proof of Theorem B.4.

$$
\begin{aligned}
\operatorname{Var}\left(\hat{T}_{i j k}\right) & =\operatorname{Var}\left(\hat{G}_{1}[i] \hat{G}_{2}[j] \hat{G}_{3}[k]\right) \\
& =\operatorname{Var}\left(\sum_{l_{1}}^{r} \sum_{l_{2}}^{r} G_{1}\left[i, l_{1}\right] G_{2}\left[j, l_{1}, l_{2}\right] G_{3}\left[k, l_{2}\right]\right) \\
& =\sum_{l_{1}}^{r} \sum_{l_{2}}^{r} \operatorname{Var}\left(\hat{G}_{1}\left[i, l_{1}\right]\right) \operatorname{Var}\left(\hat{G}_{2}\left[j, l_{1}, l_{2}\right]\right) \operatorname{Var}\left(\hat{G}_{3}\left[k, l_{2}\right]\right)+\mathbf{E}^{2}\left(\hat{G}_{1}\left[i, l_{1}\right]\right) \operatorname{Var}\left(\hat{G}_{2}\left[j, l_{1}, l_{2}\right]\right) \operatorname{Var}\left(\hat{G}_{3}\left[k, l_{2}\right]\right) \\
& +\mathbf{E}^{2}\left(\hat{G}_{2}\left[j, l_{1}, l_{2}\right]\right) \operatorname{Var}\left(\hat{G}_{1}\left[i, l_{1}\right]\right) \operatorname{Var}\left(\hat{G}_{3}\left[k, l_{2}\right]\right)+\mathbf{E}^{2}\left(\hat{G}_{3}\left[k, l_{2}\right]\right) \operatorname{Var}\left(\hat{G}_{1}\left[i, l_{1}\right]\right) \operatorname{Var}\left(\hat{G}_{2}\left[j, l_{1}, l_{2}\right]\right) \\
& +\mathbf{E}^{2}\left(\hat{G}_{1}\left[i, l_{1}\right]\right) \mathbf{E}^{2}\left(\hat{G}_{2}\left[j, l_{1}, l_{2}\right]\right) \operatorname{Var}\left(\hat{G}_{3}\left[k, l_{2}\right]\right)+\mathbf{E}^{2}\left(\hat{G}_{2}\left[j, l_{1}, l_{2}\right]\right) \mathbf{E}^{2}\left(\hat{G}_{3}\left[k, l_{2}\right]\right) \operatorname{Var}\left(\hat{G}_{1}\left[i, l_{1}\right]\right) \\
& +\mathbf{E}^{2}\left(\hat{G}_{1}\left[i, l_{1}\right]\right) \mathbf{E}^{2}\left(\hat{G}_{3}\left[k, l_{2}\right]\right) \operatorname{Var}\left(\hat{G}_{2}\left[j, l_{1}, l_{2}\right]\right) \\
& \leq \sum_{l_{1}}^{r} \sum_{l_{2}}^{r} \frac{\left\|G_{1}\right\|_{F}^{2}\left\|G_{2}\right\|_{F}^{2}\left\|G_{3}\right\|_{F}^{2}}{\left(m_{1} m_{2}\right)^{3}}+\frac{G_{1}^{2}\left[i, l_{1}\right]\left\|G_{2}\right\|_{F}^{2}\left\|G_{3}\right\|_{F}^{2}}{\left(m_{1} m_{2}\right)^{2}}+\frac{G_{2}^{2}\left[j, l_{1}, l_{2}\right]\left\|G_{1}\right\|_{F}^{2}\left\|G_{3}\right\|_{F}^{2}}{\left(m_{1} m_{2}\right)^{2}} \\
& +\frac{G_{3}^{2}\left[k, l_{2}\right]\left\|G_{2}\right\|_{F}^{2}\left\|G_{1}\right\|_{F}^{2}}{\left(m_{1} m_{2}\right)^{2}}+\frac{G_{1}^{2}\left[i, l_{1}\right] G_{2}^{2}\left[j, l_{1}, l_{2}\right]\left\|G_{3}\right\|_{F}^{2}}{m_{1} m_{2}}+\frac{G_{2}^{2}\left[j, l_{1}, l_{2}\right] G_{3}^{2}\left[k, l_{2}\right]\left\|G_{1}\right\|_{F}^{2}}{m_{1} m_{2}}+\frac{G_{1}^{2}\left[i, l_{1}\right] G_{3}^{2}\left[k, l_{2}\right]\left\|G_{2}\right\|_{F}^{2}}{m_{1} m_{2}} \\
& \leq r^{2} G_{1 a} G_{2 b} G_{3 c}\left(\frac{1}{\left(m_{1} m_{2}\right)^{3}}+\frac{3}{\left(m_{1} m_{2}\right)^{2}}+\frac{3}{m_{1} m_{2}}\right) \\
& \leq 7 r^{2}\left\|G_{1}\right\|_{F}^{2}\left\|G_{2}\right\|_{F}^{2}\left\|G_{3}\right\|_{F}^{2} \frac{1}{m_{1} m_{2}}
\end{aligned}
$$

